\title{
Suicide and Suicidal Behaviors in the Context of COVID-19 Pandemic in Bangladesh: A Systematic Review
}

\author{
Mohammed A Mamun (iD) 1,2 \\ 'CHINTA Research Bangladesh (Centre \\ for Health Innovation, Networking, \\ Training, Action and Research - \\ Bangladesh), Savar, Dhaka, 1342, \\ Bangladesh; ${ }^{2}$ Department of Public \\ Health and Informatics, Jahangirnagar \\ University, Savar, Dhaka, I342, \\ Bangladesh
}

Background: Without integration of the available information, appropriate suicide preventive actions can be hindered. Therefore, this study attempts to review the Bangladeshi COVID-19-related suicide studies for the first time.

Methods: For conducting a systematic review, the PRISMA guideline was adhered. Bangladeshi literatures concerning the COVID-19 pandemic related to either suicide case or suicidal behavior were identified within 1 to 10 April 2021, from the databases like PubMed, Scopus, PsycINFO, Web of Science, CINAHL, etc. Finally, a total of 9 literatures were included in this review.

Results: Four literatures were cross-sectional studies assessing the prevalence and risk factors of suicidal behavior, and the rest five were retrospective suicide studies concerned with either case study $(n=3)$ or case-series study $(n=2)$. The prevalence of suicidal ideation was identified to be ranging between $5 \%$ and $19.0 \%$, whereas the rate increased over time of the pandemic inception. Significant risk factors concerning suicidal behavior included the factors related to (i) socio-demographic variables: female gender, being divorced or widows or widowers or single in marital status, having lower levels of education, being urban residence, belonging to higher socioeconomic class, being unemployed, and having no children, (ii) behavior and health-related variables: lack of physical exercise, cigarette smoking, alcohol consuming, abnormal sleep status, more exposure to social media, and suffering from the higher number of physical health problems; (iii) COVID-19 pandemic-related variables: lower knowledge of COVID-19, lack of preventive COVID-19 behaviors, higher levels of COVID-19 fear, living in highly COVID-19 infected area, higher economic loss due to the pandemic, and experiencing relatives or acquaintances' death by the COVID-19; (iv) psychopathological variables: depression, anxiety, stress, insomnia, suicidal thought history, suicide attempt history, and family with a history of suicide. Conclusion: Implementing cost-effective mental health strategies along with social and community awareness for increasing help-seeking behaviors of suicide risky individuals is highly suggested.

Keywords: COVID-19 and psychological impact, pandemic and suicide, prevalence and risk factors, suicide and self-harm, suicide deaths in Bangladesh, hanging in Bangladesh, systematic review

\section{Introduction}

Along with the physical symptoms, the COVID-19 has psycho-neurological symptoms including irritability, reduced consciousness (associated with seizures), depressive mood, confusion, sleep problems, anxiety disorders, severe and rare neurological complications like brain inflammation, strokes, delirium and nerve 
damage, etc. as reported by the WHO. ${ }^{1}$ This reflects that individuals with the COVID-19 are at higher risk of mental health problems. ${ }^{2}$ Besides this, under the unexpected lockdown circumstances, general people are being reported suffering from higher levels of emotional and behavioral issues including exhaustion, detachment, irritability, poor concentration, deterioration of work performance, etc., which turns the risk of common mental health problems such as depression, insomnia, traumatic stress, anxiety disorders, etc. ${ }^{3,4}$ Adhering to the situation, $40 \%$ pooled prevalence of poor sleep quality was reported by a recent systematic review, whereas $34 \%, 26 \%, 27 \%$, and $26 \%$ prevalence was identified for psychological distress, depression, post-traumatic stress symptoms, and anxiety, respectively. $^{2}$

Suicide is the termination of psychologically vulnerable people's life. A few prior stages ought to pass before a suicide completion, which includes suicidal ideation or thought, suicide plan, suicide attempt, etc. ${ }^{5,6}$ It is estimated by the $\mathrm{WHO}^{7}$ that there are at least 20 suicide attempts for every successful suicide completion. Yearly, about 800,000 deaths are reported due to suicide, which accounts for $1.4 \%$ of all deaths globally. ${ }^{7}$ However, there is a significant link between suicide rate increment and stressful life events like viral outbreaks. Evidently, during and aftermath of the viral outbreaks (eg, 1889-1894 Russian Influenza outbreak, ${ }^{8} 1918$ influenza outbreak, ${ }^{9} 2003$ SARS epidemic, ${ }^{10}$ etc.), the suicide rate is reportedly exacerbated. For instance, the elderly suicide rate increased during the 2003 SARS epidemic in Hong Kong in 2003 and 2004, which were not suppressed to the level of the 2002 suicide rate. ${ }^{10}$

A number of studies forecasted the COVID-19 pandemic impact on suicide rate increment based on rising unemployment rates. ${ }^{11}$ To give an instance, 14.8 per 100,000 was the suicide rate in the US in 2018 , which was predicted to be 16.2 to 17.4 for $2021 .^{12}$ To combat this scenario, the rate of suicide mortality is supposed to be increased in Bangladesh, a developing country. There are no suicide surveillance systems or authorities in the country, lacking such statistics. ${ }^{13}$ Having said that, at least 14,436 people died by suicide over one year of the pandemic inception (8 March 2020 to 8 March 2021), whereas it accounts for more than $70 \%$ of the deaths occurred by the COVID-19. ${ }^{14}$ It should be noted that the country has an average trend of yearly 10,000 suicide deaths reflects that the pandemic might aggregate additional suicide occurrences. ${ }^{14,15}$ Based on the Asian Development Bank, around 9-million people were predicted to be unemployed due to the COVID-19 pandemic impact, which would lead to approximately $\$ 3$ billion GDP loss. ${ }^{16}$ As a result, accumulated mental health impacts were observed across the Bangladeshi people with financial stressors ${ }^{17}$ which eventually leads to suicidal cases. That is, early of the pandemic, within the first 3-week of April 2020, nine COVID19 related suicidality cases were reported, where all but one accounted for economic distress and sudden unemployment-related stressors. ${ }^{18,19}$

During the COVID-19 pandemic, considering the Bangladeshi people's risk to extreme psychological conditions, suicide, several studies concerned with either suicide cases or suicidal behavior were already conducted. But there is no systematic integration of information from these studies, which hinders implementing appropriate actions to reduce the unexpected suicide occurrences. Thus, for the first time, this study aims to review the Bangladeshi people's (i) suicidal behavior prevalence and risk factors based on the ecological studies and (ii) suicide stressors based on the case studies, related to the COVID-19 pandemic.

\section{Methods}

\section{Search Strategy}

Preferred Reporting Items for Systematic Reviews and Meta-Analyses (PRISMA) guideline was adhered to conduct the present systematic review ${ }^{20}$ (Figure 1). Following this guideline, PubMed was first used for searching articles systematically within 1 to 10 April 2021. In addition, other databases, including Scopus, PsycINFO, Web of Science, CINAHL, even Google Scholar and ResearchGate, were used for retrieving articles and preprints that were not indexed in PubMed. The search strategy included three types of keywords: (i) outcome of interest (suicide OR suicidal ideation OR suicide plan OR suicide attempt OR suicidal behavior OR suicidality OR self-harm); AND (ii) exposure (COVID-19 OR pandemic); AND (iii) country (Bangladesh).

\section{Study Selection Criteria}

A total of 46 articles were retrieved from several databases, where after removing the duplicate articles, 39 articles existed. Then, "Titles and Abstracts" of the retrieved articles were screened, where 19 articles were excluded for the next steps. However, a total of 20 articles" full-test was finally checked based on the inclusion criteria: (i) being a Bangladeshi study (ii) being either 

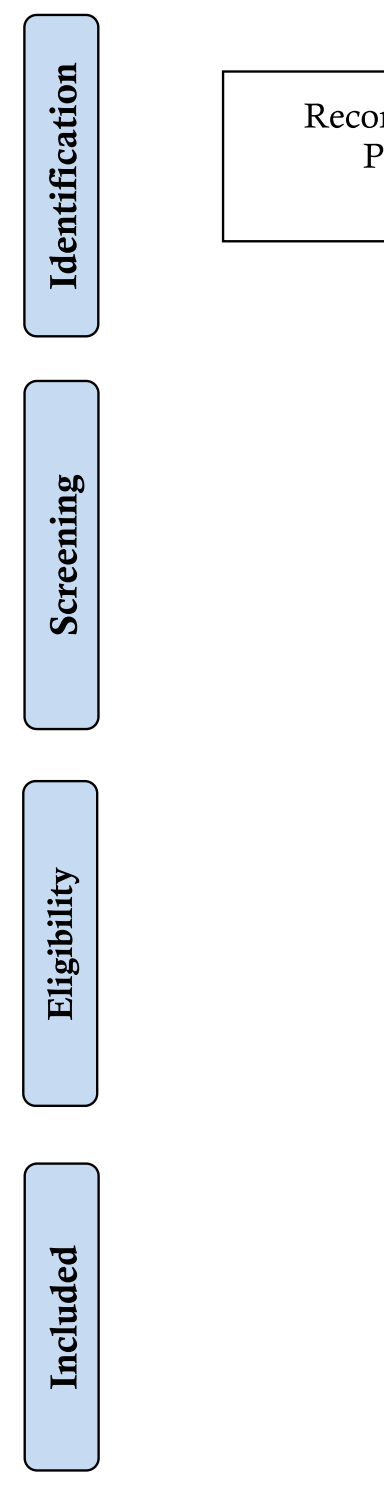

\section{Additional records identified} through other sources

$$
(\mathrm{n}=30)
$$

$$
(\mathrm{n}=16)
$$

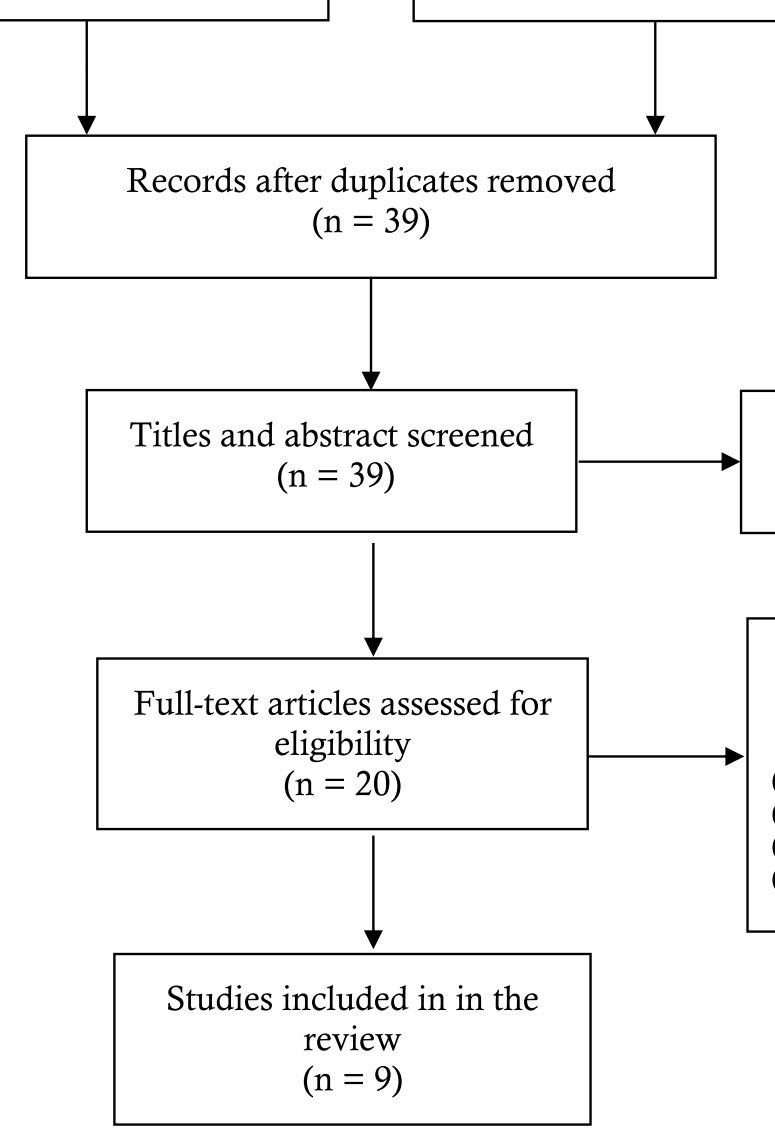

Records excluded

$(\mathrm{n}=19)$
Full-text articles excluded, with reasons $(\mathrm{n}=11)$

(i) Not Bangladeshi study $(\mathrm{n}=8)$

(ii) Not original study $(n=1)$

(iii) Data article $(\mathrm{n}=1)$

(iv) Not suicide as outcome $(\mathrm{n}=1)$

Figure I PRISMA flowchart of the present review.

case/case-series or survey-based study (iii) being related to either suicide case or suicidal behaviors (eg, suicidal ideation, suicide plan, and suicide attempt), (iv) reporting either "suicide case" or "prevalence and/or risk factors of suicidal behavior", (vi) being published in peer-reviewed journal or preprint, (vii) being published in the English language. Finally, a total of 9 articles survived in the process and were included in the present review.

\section{Data Extraction}

Two Microsoft Excel files were created to organize the information from the included studies. The data was gathered from the included survey-based studies in the first file, whereas data from the case or case-series studies were extracted in another file.

\section{Results}

\section{Description of the Included Studies}

A total of 9 articles were included in the present review after adhering to the inclusion criteria. Further, the articles are divided into two major types based on the study nature. That is, (i) survey-based study: a total of 4 cross-sectional studies assessing suicidal behaviors were retrieved (Table 1); and (ii) retrospective study: a total of 5 studies were retrieved, whereas three were case studies (reported either single suicide case or suicide-pact case) and rest 2 studies reported a series of suicide cases (Table 2). However, the cross-sectional studies were conducted from 1 April to 20 July 2020, whereas the case or caseseries studies reported the suicide cases occurring within 


\begin{tabular}{|c|c|c|c|c|}
\hline 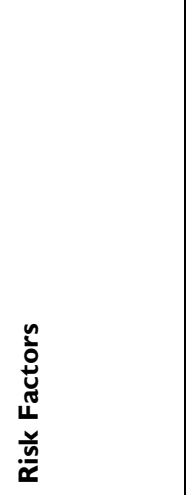 & 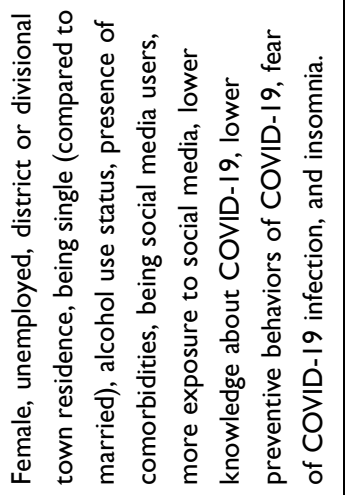 & 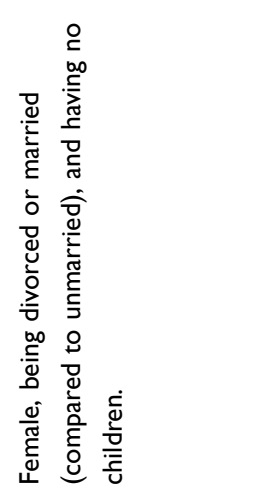 & 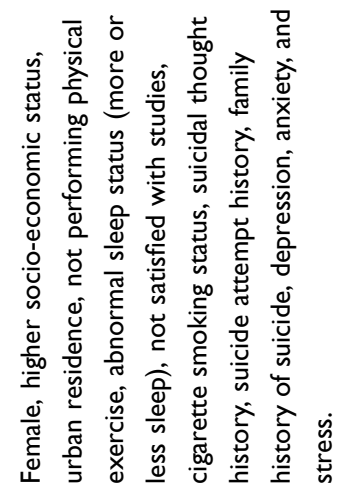 & 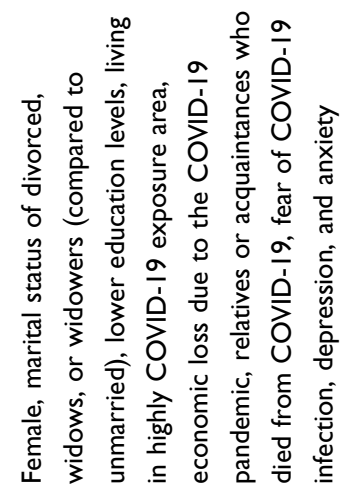 \\
\hline 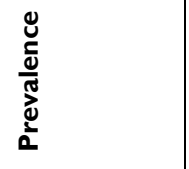 & & 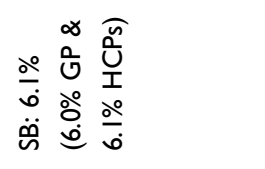 & 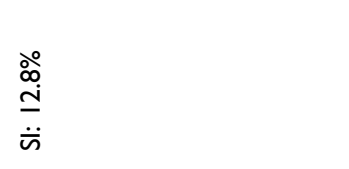 & 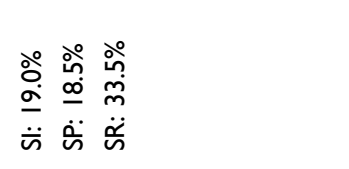 \\
\hline 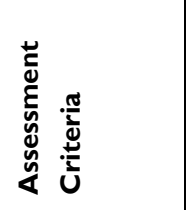 & 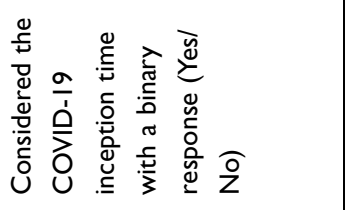 & 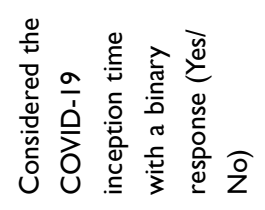 & 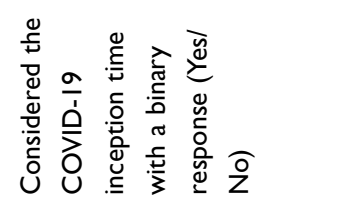 & 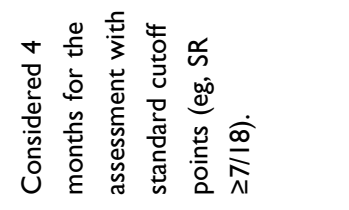 \\
\hline 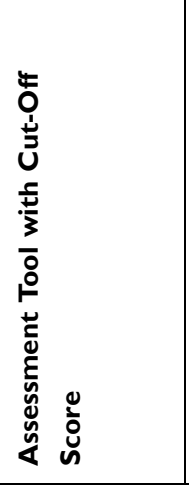 & 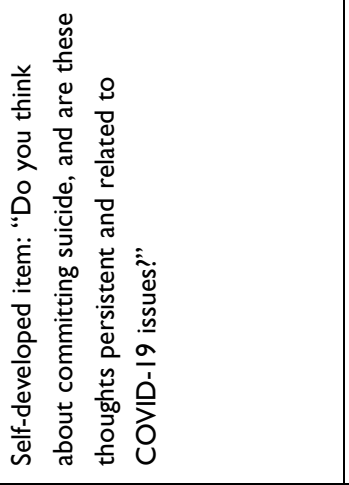 & 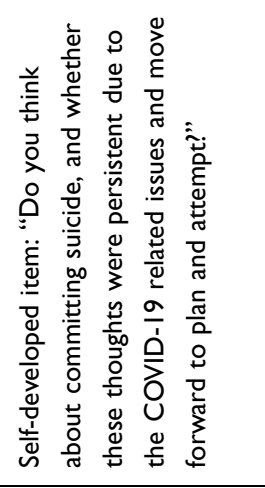 & 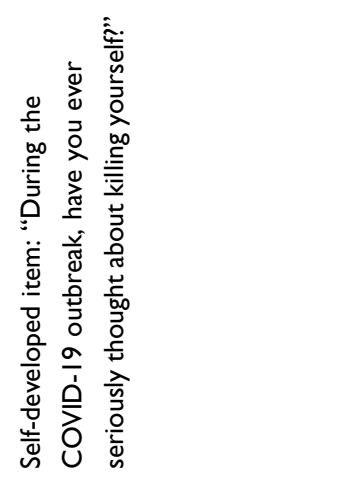 & 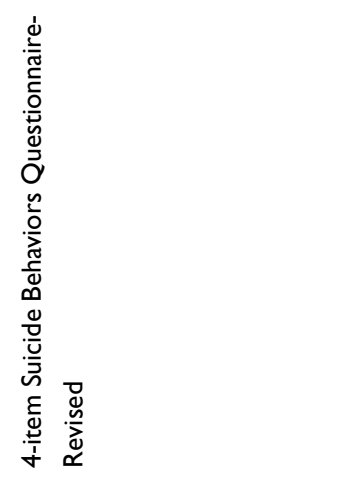 \\
\hline 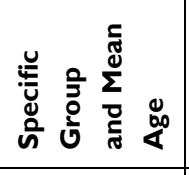 & 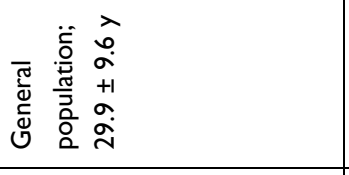 & 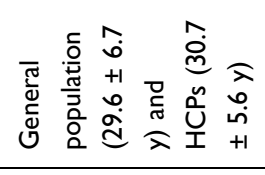 & 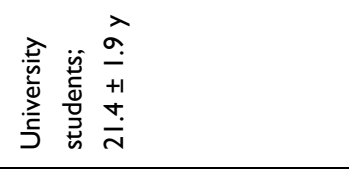 & 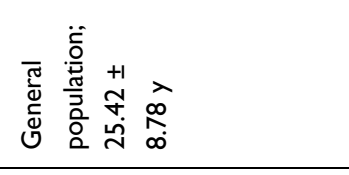 \\
\hline 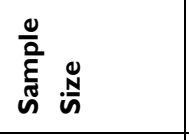 & $\begin{array}{l}\widehat{o} \\
0 \\
\text { ○ }\end{array}$ & 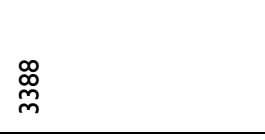 & $\underset{m}{\bar{m}}$ & $\stackrel{\underline{n}}{\bar{E}}$ \\
\hline 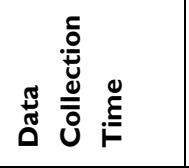 & 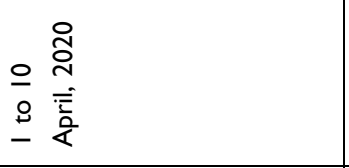 & 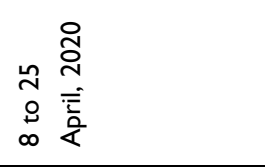 & 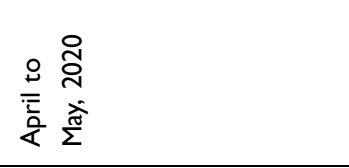 & 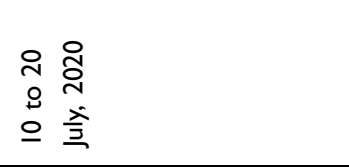 \\
\hline 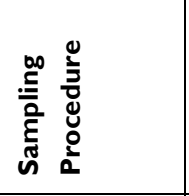 & 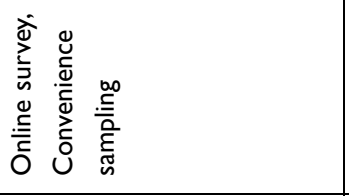 & 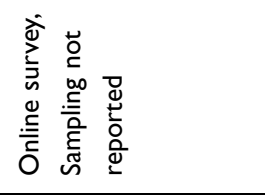 & 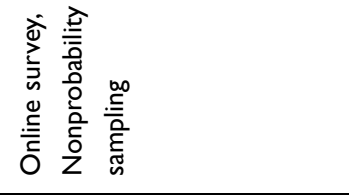 & 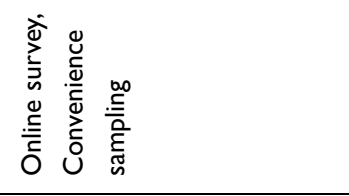 \\
\hline 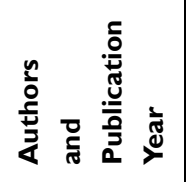 & 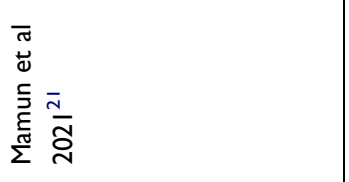 & 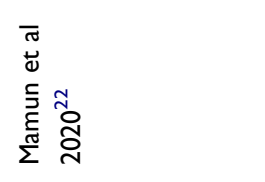 & 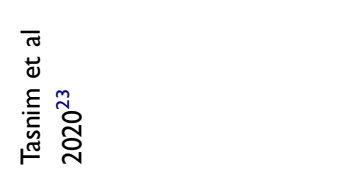 & 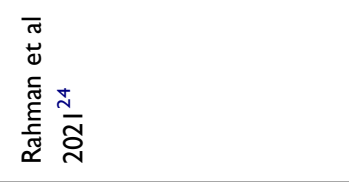 \\
\hline
\end{tabular}




\begin{tabular}{|c|c|c|c|c|c|c|c|c|c|c|c|}
\hline 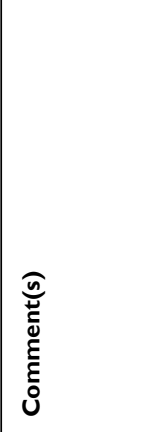 & 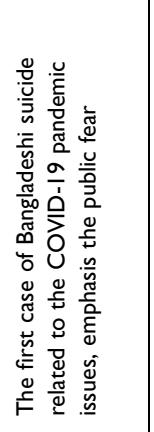 & 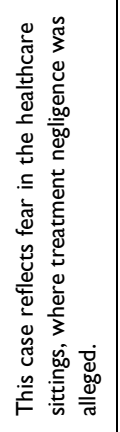 & 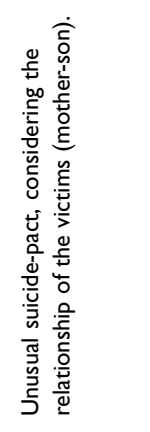 & \multicolumn{7}{|c|}{ 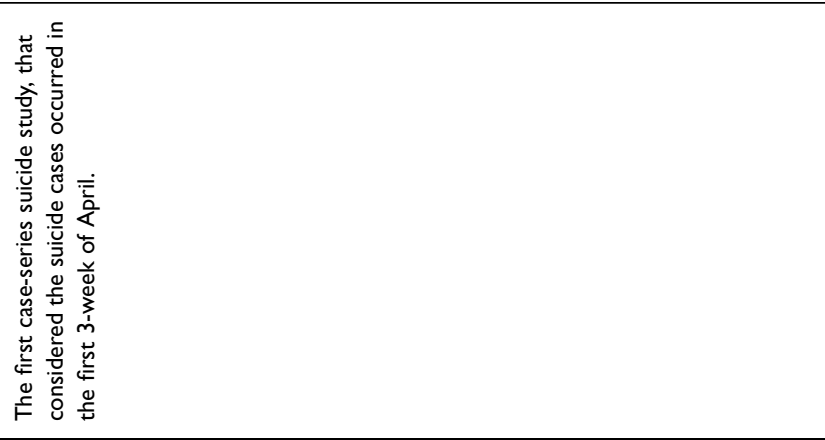 } & 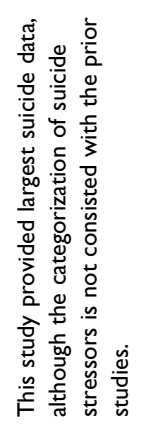 \\
\hline 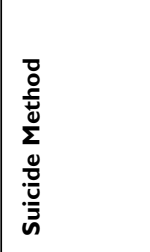 & 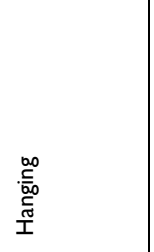 & 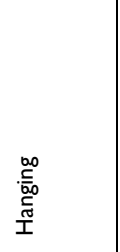 & $\begin{array}{l}\stackrel{00}{.0} \\
. \bar{c} \\
\frac{0}{0} \\
0\end{array}$ & 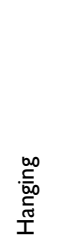 & 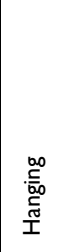 & 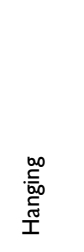 & 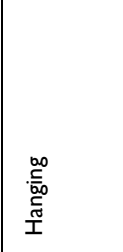 & 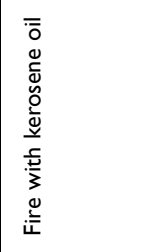 & 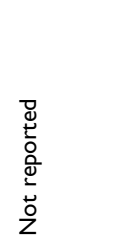 & 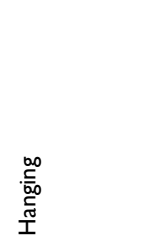 & 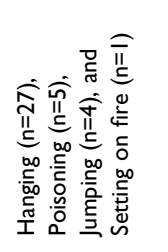 \\
\hline 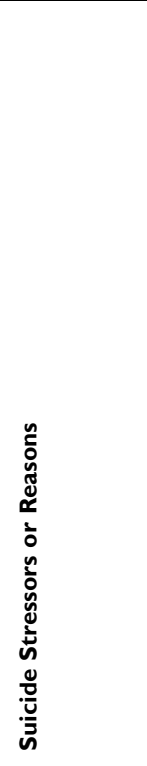 & 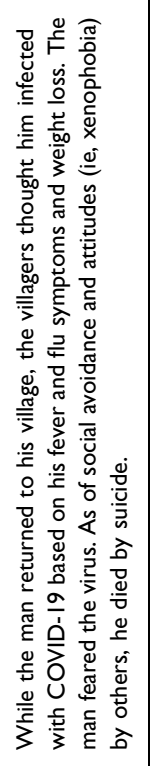 & 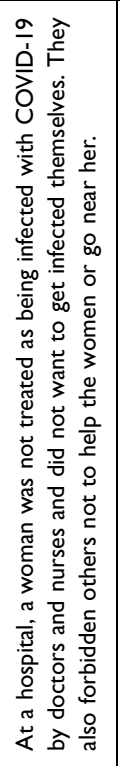 & 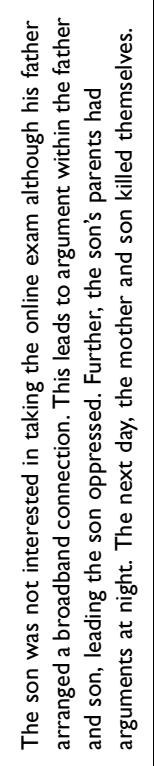 & 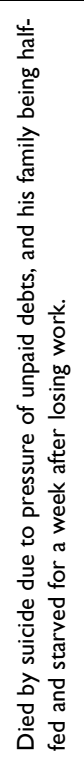 & 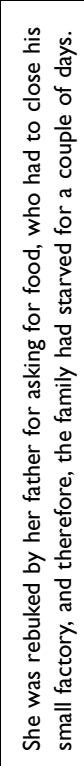 & 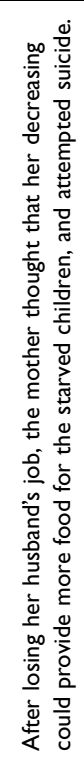 & 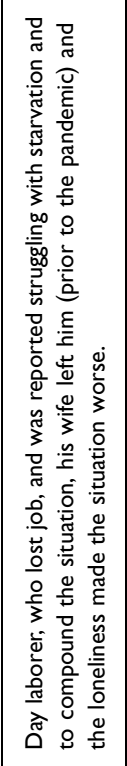 & 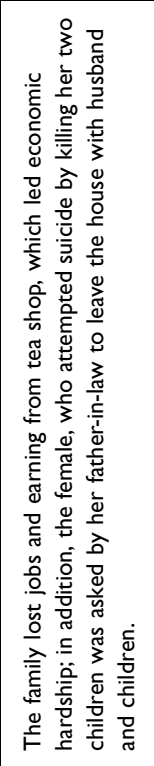 & 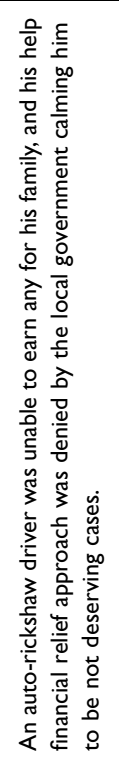 & 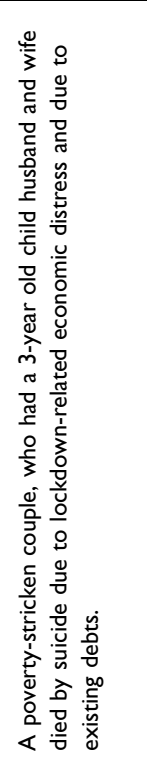 & 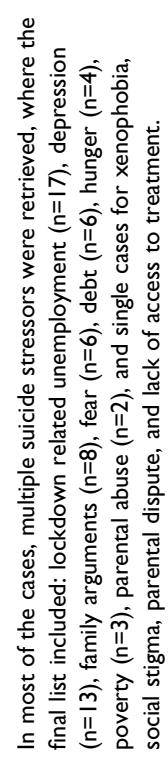 \\
\hline 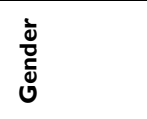 & $\frac{\frac{\omega}{\omega}}{\Sigma}$ & 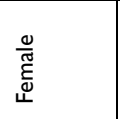 & $\begin{array}{l}\infty \\
\frac{0}{\tilde{E}} \\
\frac{0}{\tilde{\omega}} \\
\sum\end{array}$ & $\frac{\frac{\omega}{\Sigma}}{\Sigma}$ & 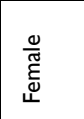 & \begin{tabular}{|l}
$\frac{0}{\tilde{E}}$ \\
$\frac{\tilde{E}}{\omega}$ \\
\end{tabular} & $\frac{\frac{\omega}{\sigma}}{\Sigma}$ & $\begin{array}{l}\frac{0}{\tilde{\Xi}} \\
\frac{\tilde{\omega}}{\mathscr{L}}\end{array}$ & $\frac{\frac{\omega}{\sigma}}{\Sigma}$ & 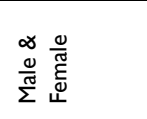 & 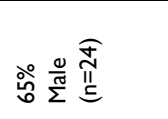 \\
\hline 离 & बे & ò & 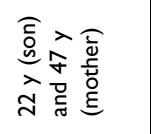 & 仓े & ô & 命 & $\hat{\lambda}$ & 蒂 & 仓े & 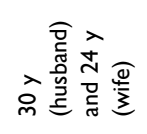 & 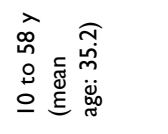 \\
\hline ป̊ & - & - & $N$ & - & - & - & - & - & - & $N$ & $\hat{m}$ \\
\hline 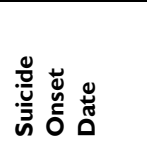 & 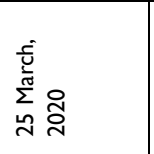 & 商苓 & 趇 & 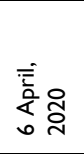 & 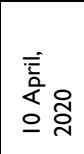 & 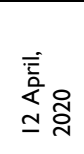 & $\begin{array}{l}\overline{\bar{E}} \\
\frac{\bar{a}}{\alpha} \\
\underline{m}\end{array}$ & 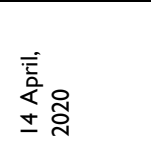 & 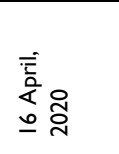 & 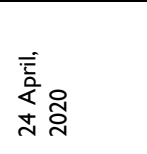 & 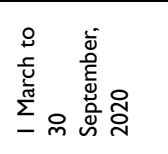 \\
\hline 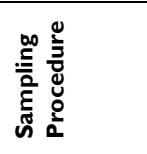 & 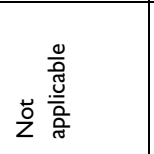 & 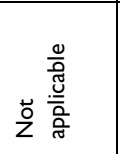 & 雚 & 惫 & & & & & & & 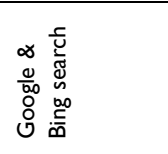 \\
\hline 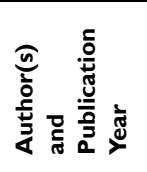 & 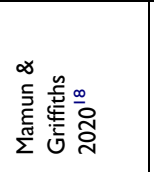 & 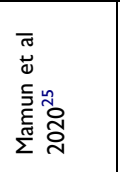 & 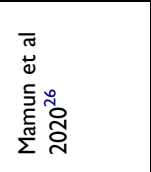 & 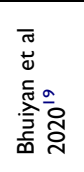 & & & & & & & 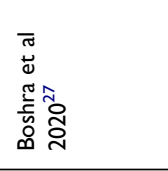 \\
\hline
\end{tabular}


25 March to 30 September 2020. The mean age of the included cross-sectional study's participants ranged between $21.4( \pm 1.9)$ to $30.7( \pm 5.6)$ years, whereas 10 to 58 years was the age range of the suicide victims as reported by the largest case-series study.

\section{Suicidal Behaviors: Prevalence Rates}

A total of 4 cross-sectional studies identified the prevalence rate of suicidal behavior, whereas the rate was assessed from the point of the COVID-19 pandemic inception in Bangladesh to survey time. First of all, Mamun et $\mathrm{al}^{21}$ compromised with the largest sample, a total of 10,067 participants, reported a 5\% prevalence of suicidal ideation based on a survey conducted within 1 to 10 April 2020. The rate increased to $6 \%$ while the survey was conducted between 8 and 25 April 2020, considering more exposure time to the lockdown situation. ${ }^{22}$ About $12.8 \%$ of suicidal ideation was reported in another study, while it was conducted from April to May $2020 .^{23}$ Finally, during the month of July, $19.0 \%$ and $18.5 \%$ of the participants reported suicidal ideation and suicide plan, respectively, where $33.5 \%$ of people at high risk of suicide were also found. ${ }^{24}$

\section{Suicidal Behaviors: Risk Factors}

All of the cross-sectional studies assessed wide-ranging risk factors related to suicidal behaviors. These factors are divided into four categories, (i) socio-demographic, (ii) behavior and health, (iii) COVID-19 pandemic, and (iv) psychopathological risk factors.

\section{Socio-Demographic Risk Factors}

Of the socio-demographic factors, the female gender was reported to significantly increase the fold of suicidal behavior in all of the included studies. ${ }^{21-24}$ Marital status was reported to be a significant risk factor in a total of 3 studies; however, heterogeneity of its risk in suicidality was also observed. For instance, 1.599 and 3.770 times higher suicidal ideation risk was observed of unmarried and divorced participants, respectively, compared to the married ones, ${ }^{22}$ consistent with Mamun et al's study. ${ }^{21}$ But compared to the unmarried participants, either divorced or widows or widowers were found being at 6.369 folds of suicidality risk in another study. ${ }^{24}$ Similarly, a lower level of education was reported increasing the risk of suicidal ideation; that is, 2.694 and 1.690 times higher risk was found for the education levels of secondary or below, and higher secondary/diploma, respectively; than the graduate or higher as education level. ${ }^{24}$ Mamun et $\mathrm{al}^{21}$ reported that suicidal ideation risk increased in a commensurate manner with the residence of the village to district and divisional cities. In contrast, another study observed that 2.34-times higher risk of suicidal ideation among the urban participants compared to rural ones. ${ }^{23}$ Regarding the socioeconomic status, only one study found a significant relationship; that is, the participants with higher socioeconomic statuses were at increased risk of suicidal ideation; ${ }^{23}$ but unemployed participants were also reported at 2-time higher risk in another study. ${ }^{21}$ In addition, having no children of the respondents reported a 1.793-fold higher risk of suicidal behavior. $^{22}$

\section{Behavior and Health-Related Risk Factors}

Unhealthy lifestyle factors such as not performing physical exercise, ${ }^{23}$ being cigarette smokers, ${ }^{23}$ and using alcohol ${ }^{21}$ were reported as being 2, 2.885, and 1.419 times higher risk of suicidal ideation. Participants reporting their sleep status to be abnormal (ie, 3.252 folds for less than normal, whereas it was 2.190 times for more than normal) were at higher risk of suicidality. ${ }^{23}$ Similarly, social media users and using it more time were independently identified as suicidal ideation risk factors. ${ }^{21}$ In addition, participants suffering from a higher number of physical health problems showed a higher risk of suicidal ideation. ${ }^{21}$

\section{COVID-I 9 Pandemic-Related Risk Factors}

Participants who reported having lower knowledge about COVID-19, lack of preventive COVID-19 behaviors, and a higher level of fear of COVID-19 infection were at higher suicidal ideation risk. ${ }^{21,24}$ Likewise, other COVID-19 related risk factors such as living in highly COVID-19 exposure area increased 1.347-times higher suicide risk, whereas 2.295 and 1.299 times higher suicide risk was identified for the participants reporting economic loss due to the COVID-19 pandemic and experiencing relatives or acquaintances' died from the COVID-19, respectively. $^{24}$

\section{Psychopathological Risk Factors}

The common psychopathological factors such as depression, anxiety and stress were the significant risk factors of suicidal ideation. That is, the risk of suicidal ideation risk was increased by $13.810,6.454$, and 9.530 times to the participants who suffered from depression, anxiety, and stress, respectively. ${ }^{23,24}$ In contrast, another study reported insomnia to be a risk factor. ${ }^{21}$ Personal history of suicidality such as suicidal thought history and suicide attempt history also increased the risk of suicidality by 7.147 and 
5.290 times. $^{23}$ Similarly, participants reported being 5.354 times highly prone to suicidal ideation if they were from a family with a history of suicidality. ${ }^{23}$

\section{Actual Suicide: Suicide Stressors Suicide Stressors Reported from Case Study}

After a few days of the COVID-19 pandemic inception in Bangladesh, on 25 March 2020, Mamun and Griffiths ${ }^{18}$ reported the first COVID-19 suicide case. The suicide victim was alleged to be decreased because of fear of being infected with the COVID-19, for which he was imposed social avoidance by the native villagers. ${ }^{18}$ In addition, a woman patient's suicide case was reported by hanging from a hospital's bathroom window grill. ${ }^{25}$ Once the woman was admitted to the hospital, nurses and doctors refused to treat her as a thought she might be infected with the virus as she had reported vomiting blood and complained of a sore throat 2-day prior to the suicide incident. Other patients were also forbidden from helping her or go near her by the healthcare staff. Finally, because of treatment negligence, the patient decreased by suicide. ${ }^{25}$ Furthermore, an unusual suicide pact considering the victims' relationship (mother and son) was reported on 11 June $2020 .^{26}$ The son was interested in taking part in the online exam, although his father arranged a broadband connection for the online exam. The conflict led to the argument between the father and son, making the son oppressed as quarreling; which led to further arguments within the son's parents at night. The next day while the man was outside for work, the mother and son killed themselves. ${ }^{26}$

\section{Suicide Stressors Reported from Case-Series Study}

The first case-series study reported a total of 8 suicidality cases (two rescued) occurring within the first three weeks of April 2020. ${ }^{19}$ All of the suicidality cases were reported because of economic and financial crisis-related issues. For instance, the victims, either being already povertystricken or losing jobs/earning sources after the lockdown imposed in Bangladesh, had to pass days with half-feeding or starving. The starving situation was visualized by the suicide of an adolescent girl, who was rebuked by her father for asking for food, while her father's small factory was closed down. In another case, a mother attempted suicide by thinking that her decrease could provide more food for the starving children because their starving faces were unbearable to her. Besides, losing the job and earning sources led to turning up familiar conflict, as reported in a case where a woman was asked to leave her father-in-law's house with her husband and children, who attempted suicide with the killing of two children. In another case, an auto-rickshaw driver who could not earn anything, and his financial relief approach was denied by the local government, calming him to be not deserving ones, committed suicide. Finally, a poverty-stricken couple, who had a 3year-old child, died by suicide due to the lockdown-related economic distress and existing debts. ${ }^{19}$

Another case-series study gathered a total of 37 suicide cases that occurred from 1 March to 30 September $2020 .{ }^{27}$ Males were predominantly dying as reported in 24 cases (out of $37 ; 65 \%$ ), whereas $64.9 \%$ and $75.7 \%$ of the victims were married and lived in a rural setting. The victims' age range was $10-58$ years, whereas 35.2 years were reported as the mean age. Multiple interrelated factors were reported attributing suicide in most of the suicide cases. For instance, unemployment usually turns to family arguments, whereas that study reported these two factors as distinct factors. However, suicide stressors were found to be lockdown related unemployment $(\mathrm{n}=17)$, depression $(n=13)$, family arguments $(n=8)$, fear $(n=6)$, debt $(n=6)$, hunger $(n=4)$, poverty $(n=3)$, parental abuse $(n=2)$, and single suicide cases for the factors including xenophobia, social stigma, parental dispute, and lack of treatment access. In the narrow view, issues related to economic and/or financial conditions appeared as the most affecting suicide stressor. ${ }^{27}$

\section{Discussion}

Due to the lockdown related to public health restricted measures, people are being reported suffering psychologically and economically, contributing to mental health problems. As a result, suicide rate increment is not unusual for a country like Bangladesh. However, the present study is the first systematic approach to review the Bangladeshi suicide and suicidal behavior-related studies concerned with the COVID-19 pandemic, which is anticipated to be helpful for the mental health policy and practice.

On 7 March 2020, Bangladesh reported the first confirmed COVID-19 case; and within a few days, the country imposed social restrictions in response to the pandemic. ${ }^{28}$ On 25 March 2020, the first COVID-19 suicide case was reported alleging fear of COVID-19 infection and social negligence or xenophobia as the suicide stressors. ${ }^{18}$ However, the first survey assessing the suicidal ideation was conducted around one month of the pandemic inception, where $5 \%$ of the prevalence rate was reported. ${ }^{21}$ The rate increased to $6 \%$ in another study while it was 
conducted a few days after the first survey-based study. ${ }^{22}$ However, about $19.0 \%$ suicidal ideation rate was found after 4-month of the pandemic inception. ${ }^{24}$ Therefore, it is evident that the more time pandemic persists in the country, the more people will be at risk of suicide.

Of the socio-demographic factors, the female gender is one of the risk factors of suicidal behavior, although they commit less suicide than males as per the WHO. ${ }^{29}$ Globally, twice times higher suicide rates were found in males (13.7 vs 7.5 per 100,000 population). ${ }^{29}$ The gender-based disparities in suicidal behavior and suicide (that is, the female has more suicidal behaviors, but less likely to commit suicide) can be attributed due to the diversities of their participating roles and responsibilities in the society, social status as well as differences in social interaction due to their biological differences. ${ }^{30,31}$ As estimated by this review, females being at higher risk of suicidal behavior, ${ }^{21-24}$ attention should be paid to ensure their mental wellbeing. However, other socio-demographic factors increasing suicidal behavior risk included being divorced or widows or widowers or single in marital status, ${ }^{21,22,24}$ having a low level of education, ${ }^{24}$ being an urban resident, ${ }^{21,23}$ belonging to higher socioeconomic status, ${ }^{23}$ being unemployed, ${ }^{21}$ having no children ${ }^{22}$ etc. It is evident that these individuals having no partner or children became more lonely as of the lockdown and social restrictions imposed, which are also reported worsening psychological wellbeing, including occurring suicide outside Bangladesh. ${ }^{4,32}$ Thus, strategies related to online engagement with friends and relatives along with having access to telepsychiatry services are highly recommended.

Participants reporting behavioral and health-related factors such as lack of physical exercise, ${ }^{23}$ cigarette smoking, ${ }^{23}$ alcohol-consuming, ${ }^{21}$ having abnormal sleep statues, ${ }^{23}$ being more exposed to social media, ${ }^{21}$ and suffering from the higher number of health problems ${ }^{21}$ were significantly prone to suicidality. These findings reflect that individuals with unhealthy lifestyles are at a higher risk of suicidal ideation, which is supposed to modify the behavioral risk factors. Consistent with the prior studies, the modifiable behaviors risk factors, lack of physical activities, for example, was found associating with either mental health issues or suicidal behaviors in a doseresponse manner. ${ }^{33}$

Also, lower knowledge of COVID-19, lack of preventive COVID-19 behaviors, higher levels of COVID-19 fear, living in highly COVID-19 infected area, experiencing economic loss due to the pandemic, and having relatives or acquaintances died from the COVID-19, etc., were the COVID-19-related suicidal ideation risk factors. ${ }^{21,24}$ Therefore, there is a need for urgent initiation of community-based health education and awareness programs, facilitation of authentic, reliable and updated information, and strict monetization of propaganda, misinformation, conspiracy theories, etc., related to the pandemic. ${ }^{34}$ These strategies can help increase public knowledge and preventive behaviors and decrease their fear regarding COVID-19; in a final turn, to reduce suicidality.

Along with these, a number of psychopathological factors (eg, depression, anxiety, stress, insomnia, suicidal thought history, suicide attempt history, and family with a history of suicide) were found to be significantly intensifying the suicidal ideation risk; ${ }^{21,23,24}$ which should be considered more critically. Because mental health problems being the proximal suicide risk factors, ${ }^{5,6}$ whereas psychological disorders attribute around $90 \%$ of the global suicide occurrences. In addition, other factors reported in this review may also play a significant role in intensifying the contribution of mental disorders to acquire more capability in suicide completion as mediators. Fear of COVID-19 infection, which was also identified as the actual suicide stressor to this review, indirectly mediated the relationship of psychological conditions (ie, depression from COVID-19 and future career anxiety) reported by a Bangladeshi study. ${ }^{35}$ Thus, the pandemic-related issues might have significant attribution in suicidality by worsening the mental health problems, which should be considered in the policy strategies.

In addition to the suicidal ideation risk factors, as found in the present review that the Bangladeshi people are mostly committing suicide due to the issues related to (i) economic crisis, (ii) fear of infection, (iii) mental health problems like depression, (iv) family arguments or conflicts, (v) parental abuse, (vi) xenophobia, (vii) social negligence, (viii) social stigma, (ix) parental dispute, (x) lack of access to treatment or treatment negligence etc. $;^{18,19,25-27}$ where economic issues included unemployment, debt, hunger, and poverty. ${ }^{19,27}$ The contributing role of economic conditions such as diminished wealth, worse economic status, and unemployment in a higher rate of suicidal behavior are reported in lower- and-middleincome countries during the normal period. ${ }^{36}$ Adhering to the situation, economic recession and job loss was also reported outside Bangladesh as the leading suicide stressors in the COVID-19 context. For instance, in Pakistan, it was reported as a prominent suicide risk attributor, ${ }^{37}$ whereas it was the second most affecting stressor after fear of COVID-19 
infection in India. ${ }^{32}$ The same Indian study also identified other stressors such as being uncomfortable in quarantine, xenophobia, social boycott, missing family and loneliness, COVID-19 work-related stress, lack of alcohol access, feeling distressed as of exam postponement, etc. ${ }^{32}$ In the prior pandemic studies, mainly four types of suicide factors were identified; that is (i) fear of being infected, (ii) being socially isolated, (iii) experiencing disruption in normal life, and (iv) experiencing the burden of long-term illness. ${ }^{10,38}$ In contrast, Bangladesh had reported most of the suicide cases concerning economic-related issues. However, it is recommended that the suicide risky stressors presented in this review should be focused on while taking mental wellbeing initiatives.

The present review can be limited because of not including the potential articles beyond the search strategies. Besides this, no quality appraisal was performed, which may limit this review. Despite these limitations, this review provides the first observation of suicide and suicidal behavior during the COVID-19 pandemic in Bangladesh, which may facilitate the implementation of mental health strategies. The strategies can be focused on the identified suicidality risk factors and actual suicide stressors, focusing on specific cohorts. In this regard, I have already provided cohort-specific detailed strategies in my prior studies, which can be referred. For instance, in the first COVID-19 suicide study, I and Griffiths ${ }^{18}$ recommended several mental health intervention programs for the general people. Similar strategies can be found in my other studies with the cohort-specific focus that includes healthcare professionals, ${ }^{25,39}$ students, ${ }^{40,41}$ economically vulnerable individuals ${ }^{37,42}$ etc.

\section{Conclusions}

Being a limited resource country, Bangladesh has the imperfect capability to fight the stressful situation turned by the COVID-19 pandemic, whereas a lack of initiatives is taken regarding mental health. In this regard, a systematic evaluation on the extreme mental health condition, suicide, is supposed to be worthy of help. Being the first review on suicide during the COVID-19 pandemic in Bangladesh, it is anticipated to have implications for mental health policymaking and practice. Considering the present review findings, the cost-effective mental health strategies with social and community awareness for increasing the suicide risky individuals' help-seeking behaviors are highly suggested.

\section{Ethics}

Ethics approval does not apply to this work.

\section{Acknowledgments}

The authors would like to acknowledge that their affiliation, the CHINTA Research Bangladesh, was formerly known as the Undergraduate Research Organization.

\section{Funding}

The present study did not get any financial support.

\section{Disclosure}

The author reported no conflicts of interest for this work.

\section{References}

1. World Health Organization. Coronavirus Disease (COVID-19); 2020. Available from: https://www.who.int/emergencies/diseases/novel-cor onavirus-2019/question-and-answers-hub/q-a-detail/coronavirus-dis ease-covid-19. Accessed December 18, 2020.

2. Krishnamoorthy Y, Nagarajan R, Saya GK, Menon V. Prevalence of psychological morbidities among general population, healthcare workers and COVID-19 patients amidst the COVID-19 pandemic: a systematic review and meta-analysis. Psychiatry Res. 2020;293:113382. doi:10.1016/j.psychres.2020.113382

3. Hossain MM, Sultana A, Purohit N. Mental health outcomes of quarantine and isolation for infection prevention: a systematic umbrella review of the global evidence. Epidemiol Health. 2020;42: e2020038. doi:10.4178/epih.e2020038

4. Brooks SK, Webster RK, Smith LE, et al. The psychological impact of quarantine and how to reduce it: rapid review of the evidence. Lancet. 2020;395(10227):912-920. doi:10.1016/S0140-6736(20) 30460-8

5. Mamun MA, Rayhan I, Akter K, Griffiths M. Prevalence and predisposing factors of suicidal ideation among the university students in Bangladesh: a single-site survey. Int J Ment Health Addict. 2020. doi:10.1007/s11469-020-00403-z

6. Klonsky ED, May AM, Saffer BY. Suicide, suicide attempts, and suicidal ideation. Annu Rev Clin Psychol. 2016;12(1)::307-330. doi:10.1146/annurev-clinpsy-021815-093204

7. World Health Organization. Mental health and substance use: suicide data; 2020. Available from: https://www.who.int/teams/mentalhealth-and-substance-use/suicide-data. Accessed February 12, 2021.

8. Smith FB. The Russian Influenza in the United Kingdom, 1889-1894. Soc Hist Med. 1995;8(1)::55-73. doi:10.1093/shm/8.1.55

9. Wasserman IM. The impact of epidemic, war, prohibition and media on suicide: United States, 1910-1920. Suicide Life Threat Behav. 1992;22::240-254. doi:10.1111/j.1943-278X.1992.tb00231.x

10. Cheung YT, Chau PH, Yip PSF. A revisit on older adults suicides and Severe Acute Respiratory Syndrome (SARS) epidemic in Hong Kong. Int J Geriatr Psychiatry. 2008;23(12)::1231-1238. doi:10.1002/gps.2056

11. John A, Okolie C, Eyles E, et al. The impact of the COVID-19 pandemic on self-harm and suicidal behaviour: a living systematic review. F1000Research. 2020;9:e1097. doi:10.12688/f1000research.25522.1

12. McIntyre RS, Lee Y. Preventing suicide in the context of the COVID19 pandemic. World Psychiatry. 2020;19(2)::250-51. doi:10.1002/ wps. 20767

13. Mamun MA, Griffiths MD. Mandatory Junior School Certificate exams and young teenage suicides in Bangladesh: a response to Arafat (2020). Int J Ment Health Addict. 2020. doi:10.1007/s11469020-00324-x

14. Dhaka Tribune. $70 \%$ more suicides than COVID-19 deaths in the last year; 2021. Available from: https://www.dhakatribune.com/bangla desh/2021/03/13/70-more-suicides-than-covid-19-deaths-in-the-lastyear. Accessed April 11, 2021. 
15. World Health Organization. Preventing suicide: a global imperative; 2014. Available from: https://apps.who.int/iris/bitstream/handle/ 10665/131056/9789241564779-ger.pdf. Accessed August 8, 2020.

16. Banna $H$ Minimising the economic impact of Coronavirus in Bangladesh. Business Standard; 2020. Available from: https://www. tbsnews.net/thoughts/minimising-economic-impact-coronavirus-ban gladesh-56449. Accessed May 13, 2020.

17. Sultana MS, Khan AH, Hossain S, et al. The association between financial hardship and mental health difficulties among adult wage earners during the COVID-19 pandemic in Bangladesh: findings from a cross-sectional analysis. PsyArXiv. 2020. doi:10.31234/osf.io/q3ehv

18. Mamun MA, Griffiths MD. First COVID-19 suicide case in Bangladesh due to fear of COVID-19 and xenophobia: possible suicide prevention strategies. Asian J Psychiatr. 2020;51:102073. doi:10.1016/j.ajp.2020.102073

19. Bhuiyan AKMI, Sakib N, Pakpour AH, Griffiths MD, Mamun MA. COVID-19-related suicides in Bangladesh due to lockdown and economic factors: case study evidence from media reports. Int $J$ Ment Health Addict. 2020. doi:10.1007/s11469-020-00307-y

20. Moher D, Liberati A, Tetzlaff J, Altman DG. Preferred reporting items for systematic reviews and meta-analyses: the PRISMA statement. Int J Surg. 2010;8(5)::336-341. doi:10.1016/j.ijsu.2010.02.007

21. Mamun MA, Sakib N, Gozal D, et al. The COVID-19 pandemic and serious psychological consequences in Bangladesh: a populationbased nationwide study. $J$ Affect Disord. 2021;279::462-472. doi:10.1016/j.jad.2020.10.036

22. Mamun MA, Akter T, Zohra F, et al. Prevalence and risk factors of COVID-19 suicidal behavior in Bangladeshi population: are healthcare professionals at greater risk? Heliyon. 2020;6(10):e05259. doi:10.1016/j.heliyon.2020.e05259

23. Tasnim R, Islam MS, Sujan MSH, Sikder MT, Potenza MN. Suicidal ideation among Bangladeshi university students early during the COVID-19 pandemic: prevalence estimates and correlates. Child Youth Serv Rev. 2020;119:105703. doi:10.1016/j. childyouth.2020.105703

24. Rahman ME, Zubayer AA, Al mazid bhuiyan MR, Jobe MC, Khan MKA. Suicidal behaviors and suicide risk among Bangladeshi people during the COVID-19 pandemic: an online cross-sectional survey. Heliyon. 2021;7(2):e05937. doi:10.1016/j.heliyon.2021.e05937

25. Mamun MA, Bodrud-Doza M, Griffiths MD. Hospital suicide due to non-treatment by healthcare staff fearing COVID-19 infection in Bangladesh? Asian J Psychiatr. 2020;54:102295. doi:10.1016/j. ajp.2020.102295

26. Mamun MA, Chandrima RM, Griffiths MD. Mother and son suicide pact due to COVID-19-related online learning issues in Bangladesh: an unusual case report. Int J Ment Health Addict. 2020. doi:10.1007/ s11469-020-00362-5

27. Boshra SN, Islam MM, Griffiths MD. The demography and apparent risk factors of COVID-19-related suicides in Bangladesh in a sevenmonth period of the pandemic. medRxiv. 2020. doi:10.1101/ 2020.08.11.20171272

28. Anwar S, Nasrullah M, Hosen MJ. COVID-19 and Bangladesh: challenges and how to address them. Front Public Heal. 2020;8:154. doi:10.3389/fpubh.2020.00154

Psychology Research and Behavior Management

\section{Publish your work in this journal}

Psychology Research and Behavior Management is an international, peer-reviewed, open access journal focusing on the science of psychology and its application in behavior management to develop improved outcomes in the clinical, educational, sports and business arenas. Specific topics covered in the journal include: Neuroscience, memory and decision making; Behavior modification and management; Clinical
29. World Health Organization. Suicide in the world: global health estimates; 2019. Available from: https://apps.who.int/iris/bitstream/han dle/10665/326948/WHO-MSD-MER-19.3-eng.pdf? Accessed May 10,2021 .

30. Göktaş S, Metintaş S. Suicide mortality trends by age, gender and method in Turkey, 2002-2015. Türkiye Halk Sagligi Derg. 2019;17::195-206. doi:10.20518/tjph.442055

31. Vijayakumar L. Suicide in women. Indian J Psychiatry. 2015;57(6): S233-38. doi:10.4103/0019-5545.161484

32. Dsouza DD, Quadros S, Hyderabadwala ZJ, Mamun MA. Aggregated COVID-19 suicide incidences in India: fear of COVID19 infection is the prominent causative factor. Psychiatry Res. 2020;290:113145. doi:10.1016/j.psychres.2020.113145

33. Grasdalsmoen M, Eriksen HR, Lønning KJ, Sivertsen B. Physical exercise, mental health problems, and suicide attempts in university students. BMC Psychiatry. 2020;20(1):175. doi:10.1186/s12888-02002583-3

34. Hasan M, Hossain S, Saran T, Ahmed H. Addressing the COVID-19 related stigma and discrimination: a fight against "infodemic" in Bangladesh. Minerva Psichiatr. 2020;61(4):184-187. doi:10.23736/ S0391-1772.20.02088-9

35. Mahmud MS, Talukder MU, Rahman SM. Does "Fear of COVID19 " trigger future career anxiety? An empirical investigation considering depression from COVID-19 as a mediator. Int J Soc Psychiatry. 2020;002076402093548. doi:10.1177/0020764020935488

36. Iemmi V, Bantjes J, Coast E, et al. Suicide and poverty in low-income and middle-income countries: a systematic review. Lancet Psychiatry. 2016;3(8)::774-783. doi:10.1016/S2215-0366(16)30066-9

37. Mamun MA, Ullah I. COVID-19 suicides in Pakistan, dying off not COVID-19 fear but poverty? The forthcoming economic challenges for a developing country. Brain Behav Immun. 2020;87::163-166. doi:10.1016/j.bbi.2020.05.028

38. Yip PSF, Cheung YT, Chau PH, Law YW. The impact of epidemic outbreak: the case of severe acute respiratory syndrome (SARS) and suicide among older adults in Hong Kong. Cris J Cris Interv Suicide Prev. 2010;31(2)::86-92. doi:10.1027/0227-5910/a000015

39. Jahan I, Ullah I, Griffiths MD, Mamun MA. COVID-19 suicide and its causative factors among the healthcare professionals: case study evidence from press reports. Perspect Psychiatr Care. 2021. doi:10.1111/ppc.12739

40. Manzar MD, Albougami A, Usman N, Mamun MA. Suicide among adolescents and youths during the COVID-19 pandemic lockdowns: a press media reports-based exploratory study. $J$ Child Adolesc Psychiatr Nurs. 2021;34(2)::139-146. doi:10.1111/jcap.12313

41. Mamun F, Hosen I, Misti JM, Kaggwa MM, Mamun MA. Mental disorders of Bangladeshi students during the COVID-19 pandemic: a systematic review. Psychol Res Behav Manag. 2021;14:645-654. doi:10.2147/PRBM.S315961

42. Mamun MA. The first COVID-19 triadic (homicide!)-suicide pact: do economic distress, disability, sickness, and treatment negligence matter? Perspect Psychiatr Care. 2020. doi:10.1111/ppc. 12686 applications; Business and sports performance management; Social and developmental studies; Animal studies. The manuscript management system is completely online and includes a very quick and fair peer-review system, which is all easy to use. Visit http://www. dovepress.com/testimonials.php to read real quotes from published authors. 